\title{
Doctor-Patient Cancer Screening Communications among Church-based Chinese Adults - The Role of Caregiver Experience and Family History
}

\author{
Su-I Hou*
}

\begin{abstract}
Objective: Significant gap exists in the literature examining cancer screening communication related factors among Chinese immigrants. This study examined the role of cancer caregiver experience and family history on doctor-patient cancer screening communication among church-based Chinese adults. Methods: A self-administered survey was conducted among adults from 9 Chinese churches $(n=372)$. Cancer Communication was measured by "Dr. recommended screenings" and "Talked to doctors about cancer screenings". The survey was developed in English and translated in Chinese. Results: Mean age was 44.31 ( $\mathrm{SD}=14.74), 60 \%$ were males, 72\% were married, majority had college education (85\%), and $17 \%$ reported had been a primary cancer caregiver and $54 \%$ reported having family cancer history. Cancer caregivers scored higher on doctor-patient cancer communication, as well as cancer knowledge and screening norms. Participants with family cancer history were also more likely to talk to doctor about screening, as well as perceived higher cancer risk, lower health status, and screening barriers. Multiple regression analyses showed that primary caregiver experience was still a significant predictor on talking to doctors about cancer screenings $(\mathrm{OR}=2.1 ; 95 \% \mathrm{CI}=[1.10,4.01] ; \mathrm{p}=0.025)$, yet doctors more like to recommend screening among caregivers became non-significant. The significant influence of family cancer history on talking with doctors on cancer screenings also disappeared. Older age $(\mathrm{OR}=2.52 ; \mathrm{p}=0.006)$ and being married $(\mathrm{OR}=2.45 ; \mathrm{p}=0.022)$ were significant on predicting communication of cancer screenings with doctors. Data also showed that doctors were more likely to recommend cancer screenings to older $(\mathrm{OR}=2.75, \mathrm{p}<.001)$, married $(\mathrm{OR}=2.57 ; \mathrm{p}=0.006)$ adults. Conclusion: Current study calls attentions to primary cancer caregiver experience, family history, age, and marital factors when designing tailored doctor-patient cancer screening communication programs among church-based Chinese to address cancer disparities.
\end{abstract}

Keywords: Cancer screening communication- Chinese- caregiver- family history

Asian Pac J Cancer Prev, 22 (1), 241-247

\section{Introduction}

Rationales for conducting cancer communication study in Chinese Immigrants

Chinese-American is the largest Asian subpopulation (23.0\%), with over two-thirds as first-generation immigrants born overseas (ACS, 2016). However, current research show Chinese-American is under-represented in cancer studies and reported low screening adherence. With complex barriers towards cultural, language, and healthcare access, Chinese immigrants face deepened disparities in cancer screening participations (Sentell et al., 2015; Hou, 2010). Faith-based organization has a unique position to deliver health and social services to the hard-to-reach communities (Hou and Cao, 2018a; Hou and Liu, 2018b; Hou, 2016a; Hou, 2016b; Holt et al, 2009). However, there is a significant gap in the literature examining Chinese or Chinese-American in faith-based organizations overall. Majority of the existing faith-based cancer interventions have been conducted among African Americans (Griffith et al, 2012; Emerson et al., 2009; Holt et al., 2009) and Latino Americans (Schwingel and Galvez, 2016; Allen et al, 2014; Colon-Otero et al., 2014), yet few have focused among Asian immigrants overall (Hou and Cao, 2019; Hou and Cao, 2018a). Significant gap also exists in the literature examining doctor-patient cancer screening communication among Chinese immigrants.

The role of primary cancer caregiver experience on doctor-patient cancer screening communication

Limited studies have examined doctor-patient cancer communication among cancer caregivers and results have been inconsistent. Studies show while caregivers had higher cancer awareness (Elangovan et al., 2016), caregivers reported having limited cancer knowledge and very low screening self-efficacy (Nasiriani, et al., 2017; Anusha and Nagarathnam, 2016). A study in Iran 
found only $13 \%$ of the caregivers were screened for mammography, over $70 \%$ of those who were not screened indicated "the doctor did not prescribe for me" as the main reason, and near $90 \%$ did not know when or how to perform breast self-exam (Naririani et al., 2017).

There has been overwhelming evidence showing provider recommendation significantly improves screening rates (Peterson et al., 2016). Family cancer caregivers desire cancer information and communication with doctors (Hou 2016a; Longacre et al., 2015). However, studies on cancer communication with doctors examining the role of caregivers have been limited, either within the U.S. or overseas. The few existing studies show primary care physicians are not recommending screenings among caregivers at a satisfactory level, and a significant portion of caregivers had at least one or more unanswered screening concerns (Lin et al., 2016).

The role offamily cancer history on doctor-patient cancer screening communication

The link between family cancer history and higher cancer risk has been confirmed by scientists and researchers (CDC, 2019; Ramsey et al., 2006). However, existing studies find those with family cancer history or high risk of cancers are still uninformed of their cancer risk or never received a physician recommendation for screening (Salimzadeh et al., 2016; Fletcher et al., 2007). A large U.S. colorectal cancer study among patients with family history showed almost half did not know they should be screened at a younger age (Fletcher et al., 2007). Similarly, another large colorectal cancer study in Iran examined first degree relatives (FDRs) of cancer patients also showed low screening rate and knowledge, and low awareness of their own increased cancer risk (Salimzadeh et al., 2016). These unsatisfactory findings may at least partially due to the fact that general physicians need to be educated to better perform their gatekeeper role and answer screening related questions among patients with family cancer history (Lunsford et al., 2018; Rose et al., 2001). Rose and colleague studied physician's knowledge on referring patients with family history of cancers showed that primary care doctors have difficulty in deciding whether to refer due to limited genetic knowledge. Most general physicians prefer referring only moderate and high risk, but not low risk patients for screenings (Rose et al., 2001).

Gap on the role of caregiver and family history on doctorpatient cancer screening communications among Chinese adults

Although there have been a number of studies focusing on the mental or physical health among cancer caregivers (Warapornmongkholkul et al., 2018; Lee et al., 2017; Nipp et al., 2016; Sklenarova et al., 2015). There are limited studies examining doctor-patient cancer screenings communication in light of the role of caregivers or those with family history (Polek and Hardie, 2016). Existing limited literature pointed while caregivers might view cancers less fatalistic, their overall cancer knowledge and communication are low. Doctor-patient cancer screening communication deserves greater attention, especially among the under-studied Chinese or Chinese immigrant groups. The current study examines the role of cancer caregiving experience and family cancer history on doctor-patient screening communication among church-based Chinese adults. The author hypothesizes that primary cancer caregivers and those with family history will be more likely to have doctor-patient cancer communication, as well as score higher on cancer knowledge and beliefs. Given a lack of literature focusing on doctor-patient cancer screening communication and the role of caregiver and family history, and with the strength of a mixed sample of Chinese-immigrants in the U.S. and overseas, this study fills in the important gaps in the literature on both the subjects and populations, as well as providing critical implications to healthcare professionals interacting with Chinese patients.

\section{Materials and Methods}

\section{Design and Sample}

The current study was a cross-sectional study assessing doctor-patient cancer screening communication and examining the role of caregiver and family history among church-going Chinese adults. A convenience sample of five major Chinese churches in a large metropolitan city in the Southeastern U.S., and four Chinese churches in a large metropolitan city in Taiwan participated in the study (total n=372) (Hou and Cao, 2019; Hou and Liu, 2018b). This convenient sample was chosen given existing established relationship with the researcher. Strategies used to recruit study participants included informing key church leaders, making formal announcements at the Sunday services, setting up tables in high-traffic areas such as the sanctuary foyer, lobby, cafeteria, as well as visiting small group gatherings. About $80 \%$ of the Chinese adults invited voluntarily participated $(n=372)$. All the study churches provided mandarin services as their main services. Reasons for possible non-participation could be due to lack of time or interests, or other obligations at the time of the survey. The study was approved by the human subject office at the investigator's institution.

In order to detect meaningful difference on doctor-patient cancer communication, as well as cancer knowledge and beliefs, by caregiver experience (yes / no) or family cancer history (yes / no), minimum sample size needed were calculated. Mean differences and standard deviations were estimated from earlier published results (Hou and Cao, 2019; Hou and Liu, 2018b), using 5\% alpha level, $80 \%$ detecting power, and 1:1 ratio between two groups, minimum sample sizes ranged between 21 to 63 per group in these comparisons. The current study sample met the minimum sample size to have $80 \%$ power to detect meaningful differences between groups.

\section{Measurement}

Study questionnaire was developed in English, translated into Chinese, and back-translated to ensure consistencies and accuracy of item meanings (Hou et al., 2014; Hou and Chen, 2004; Hou et al., 2003). The self-administered survey took 10-15 minutes to complete. A small thank-you gift was provided for each participant. 
Cancer communications were measured by two self-reported survey items, "Dr. recommended screenings" and "Talked to doctors about cancer screenings" to capture the two-way communication dimensions. Both the cancer caregiver experience and family cancer history were yes / no questions asking whether participants had "ever been a primary caregiver for a family member", and whether participants "had any relatives or family members who had been diagnosed with cancers."

Author's 8-item validated Cancer Screening Knowledge Test (CSKT) (Hou and Liu, 2018b; Hou et al., 2014; Hou, 2016c) and a 14-item Cancer Warning Signs Test (CWST) (Hou and Liu, 2018b) were used to assess objective cancer knowledge. Both scales were developed in collaboration with community partners and stakeholders, as well as pilot tested among multiple Chinse samples in Taiwan with satisfactory reliabilities and validities (Hou and Liu, 2018b; Hou, 2016c; Hou et al., 2014). The Cronbach alphas of CSKT and CWST scales were .701 and .909 respectively. CSKT consisted of a good mix of eight (8) easy and moderate difficult items measuring key cancer risk factors, need for regular screenings, recommended guidelines of major ageappropriate cancer screenings, and cancer early signs. The CWST measured the seven (7) cancer warning signs (C.A.U.T.I.O.N.) endorsed by the World Health Organization, as well as seven (7) non-cancer warning signs (Hou, 2016c). Each knowledge item answered correctly was coded as " 1, , and coded as " 0 " otherwise. Possible scale ranges were $0-8$ for CSKT and $0-14$ for CWST. One research-tested subjective cancer knowledge item using 5-point Liker scale was used to measure participants' subjective cancer knowledge level (Hou and Liu, 2018b; Hou, 2016c; Hou et al., 2014). The subjective knowledge item was a five-point liker scale item, with 5 being very high and 1 being very low.

A previously validated theory-based Cancer Screening Belief Scale (CSBS) was also used in the current study (Hou, 2007). The domains for the CSBS include 3-item perceived screening benefits (pros), 4-item perceived screening barriers (cons), and 3-item perceived norms (norms). The reliabilities of these sub-scales were satisfactory with Cronbach alphas of 0.818 (CITC range, 0.652 to 0.689 ) for CSBS-Pros, 0.649 (CITC range, 0.343 to 0.497 ) for CSBS-Cons, and 0.722 (CITC range,
0.411 to 0.648 ) for CSBS-Norms (Hou, 2007). All items were measured via 5-point Likert scales. Possible ranges were 1-15 for CSBS-pros and CSBS-norms, and 1-20 for CSBS-cons. This scale has been tested among multiple Chinese sample with consistent satisfactory evidence of validities and reliabilities (Hou and Liu, 2018b; Hou, 2016a; 2016b; 2016c; Hou et al., 2014).

Analyses

Descriptive analyses were used for demographic variables. Chi-square tests were used to compare the two cancer communication variables by primary cancer caregiver and family cancer history. T-tests were used to compare continuous variables including subjective and objective cancer knowledges, cancer screening beliefs scales between groups. Multiple logistic regressions were used to exam how caregiver experience and family cancer history predicted the two cancer communications variables, adjusting for demographics co-variables including age, gender, marital status, education, and country.

\section{Results}

\section{Participant characteristics}

A total of 372 Chinese adults from nine churches participated. About half of the participants lived in the U.S and half lived in Taiwan. Overall $62 \%$ of the participants aged $40+$ years, $60 \%$ were males, $72 \%$ married, and majority had college education (85\%). Overall 17\% reported had been a primary caner caregiver, and over half of the study participants reported family cancer history (54\%). There were no significant differences between country sites by age, marital status, family cancer history, or talk to doctor about screenings (Hou and Cao, 2019).

Cancer communications, knowledge, and screening beliefs by cancer caregiver experience

Study also found that older (49.9 vs. 43.2 years; $\mathrm{p}=.002)$ and male Chinese adults $(73.3 \%$ vs. $57.0 \%$; $<<.05)$ were more likely to be cancer caregivers. Results showed that those who had been cancer caregivers were also more likely to talk with doctors about cancer screenings (48.3\% vs. $23.4 \%, \mathrm{p}<.001)$ and report doctors recommended cancer screenings to them $(56.7 \%$ vs. $31.8 \%, \mathrm{p}<.001)$.

Table 1. Cancer Communications, Knowledge, \& Screening Beliefs by Primary Cancer Caregiver Experience

\begin{tabular}{|c|c|c|c|}
\hline Variables & $\begin{array}{c}\text { Had been a primary Caregiver } \\
(\mathrm{n}=63) \\
\text { Mean }(\mathrm{SD})\end{array}$ & $\begin{array}{l}\text { Not a primary cancer } \\
\text { Caregiver }(\mathrm{n}=309) \\
\text { Mean (SD) }\end{array}$ & p-value \\
\hline Dr. recommend screenings & $56.70 \%$ & $31.80 \%$ & $<0.001 * *$ \\
\hline Talked with dr. on screenings & $48.30 \%$ & $23.40 \%$ & $<0.001 * *$ \\
\hline Subjective cancer knowledge (1-item) & $3.33(.951)$ & $2.73(.906)$ & $<0.001 * *$ \\
\hline Cancer Screening Knowledge Test (8-item) & $6.16(1.089)$ & $5.78(1.2589)$ & $0.030^{*}$ \\
\hline Cancer Warning Sign Test (14-item) & $6.95(3.070)$ & $5.97(3.189)$ & $0.036^{*}$ \\
\hline CSBS-Pros (3-items) & $12.92(2.136)$ & $12.44(2.096)$ & 0.11 \\
\hline CSBS-Cons (4-items) & $10.53(3.365)$ & $10.81(2.874)$ & 0.515 \\
\hline CSBS-Norms (3-items) & $11.95(1.861)$ & $11.12(2.058)$ & $0.004 *$ \\
\hline
\end{tabular}

CSBS, Cancer Screening Belief Scale (CSBS) 
Table 2. Cancer Communications, Knowledge, \& Screening Beliefs by Family Cancer History

\begin{tabular}{lccc}
\hline Variables & $\begin{array}{c}\text { Had Family History (n=201) } \\
\text { Mean (SD) }\end{array}$ & $\begin{array}{c}\text { No Family History (n=171) } \\
\text { Mean (SD) }\end{array}$ & p-value \\
\hline Dr. recommend screenings & $39.70 \%$ & $30.70 \%$ & 0.079 \\
Talked with dr. on screenings & $32.20 \%$ & $21.50 \%$ & $0.025^{*}$ \\
Subjective cancer knowledge (1-item) & $2.91(.914)$ & $2.73(.953)$ & 0.061 \\
Cancer Screening Knowledge Test (8-item) & $5.89(1.435)$ & $5.74(1.654)$ & 0.36 \\
Cancer Warning Sign Test (14-item) & $6.43(3.186)$ & $5.70(3.157)$ & $0.034^{*}$ \\
CSBS-Pros (3-items) & $12.59(2.106)$ & $12.43(2.098)$ & 0.467 \\
CSBS-Cons (4-items) & $10.40(2.963)$ & $11.19(2.882)$ & $0.011^{*}$ \\
CSBS-Norms (3-items) & $11.43(1.807)$ & $11.03(2.287)$ & 0.072 \\
\hline
\end{tabular}

Analyses also showed that those who had been a primary cancer caregiver to a family member were more likely to score higher on perceived cancer knowledge (3.33 vs. $2.73, \mathrm{p}<.001)$, cancer screening knowledge test (6.16 vs. $5.78, \mathrm{p}=.030)$, cancer warning sign test (6.95 vs. 5.97, $\mathrm{p}=.036)$, and perceived cancer screening norms $(11.95$ vs. 11.12, $\mathrm{p}=.004)$.

Cancer communications, knowledge, and screening beliefs by family cancer history

Current data show that Chinese adults with family cancer history tended to be older ( 46.4 vs. 41.9 years; $\mathrm{p}=.004)$ and married (78.8\% vs. $64.1 \% ; \mathrm{p}=.002)$. Findings showed that participants with family cancer history were more likely to report talked with doctors on cancer screenings (32.2\% vs $21.5 \%$; $=.025)$, and scored higher on cancer warning sign tests $(6.43$ vs. $5.70 ; .034)$ and lower on perceived cancer screening barriers (10.40 vs. $11.19, \mathrm{p}=.011)$. Data also showed those with family cancer history were more likely to perceived higher cancer risk (2.78 vs. $2.53 ; p=.003)$ and lower health status $(3.14$ vs. 3.46; $\mathrm{p}=.001)$. Although findings indicated doctors were also more likely to recommend those with family cancer histories to screen for cancers, the difference was not statistically significant $(39.7 \%$ vs $30.7 ; p=.079)$. There wer, however, no significant differences on doctors recommending screenings, subjective cancer and screening knowledge by family cancer history.

Multiple logistic regression analyses

After controlling for demographics (age, gender, marital, and education) along with country difference
(USA vs. Taiwan), multiple logistic regression analyses showed that primary caregiver experience to a family member was still a significant predictor on talking to doctors about cancer screenings $(\mathrm{OR}=2.1 ; 95 \% \mathrm{CI}=[1.10$, 4.01]; $\mathrm{p}=.025)$, yet doctors more like to recommend screening among caregivers became non-significant. The significant influence of family cancer history on talking with doctors on cancer screenings also disappeared after controlling for key demographics and country. Oder age $(\mathrm{OR}=2.52 ; \mathrm{p}=.006)$ and being married $(\mathrm{OR}=2.45 ; \mathrm{p}=.022)$ were also significant on predicting communication of cancer screenings with doctors. Data also showed that doctors were more likely to recommend cancer screenings to older $(\mathrm{OR}=2.75, \mathrm{p}<.001)$, married $(\mathrm{OR}=2.57 ; \mathrm{p}=.006)$ adults. There were no significant differences on cancer communications by gender or education.

\section{Discussion}

Two previously published articles also analyzed data from the current study sample (Hou and Cao, 2019; Hou and Liu, 2018b). One focused on objective versus subjective knowledge by younger versus older age groups (Hou and Liu, 2018b). The other one compared cancer knowledge and beliefs among participants from different country, USA vs. Taiwan (Hou and Cao, 2019). To provide deeper insights on the complex factors critical for tailored cancer screening communication intervention development, this study conducted further analyses to examine the role of primary caregiver experience and family cancer history on doctor-patient cancer screening

Table 3. Multiple Regressions of Caregiver Experience and Family Cancer History on Cancer Communications, Adjusting for Demographics (Age, Gender, Marital, and Education) and Country

\begin{tabular}{|c|c|c|c|c|}
\hline \multirow{3}{*}{$\begin{array}{l}\text { DV } \\
\text { IV }\end{array}$} & \multirow{2}{*}{\multicolumn{2}{|c|}{$\begin{array}{c}\text { Dr. recommend screenings } \\
\mathrm{X}_{(7)}^{2}=52.89(\mathrm{p}<.001) ; 67.8 \%\end{array}$}} & \multirow{2}{*}{\multicolumn{2}{|c|}{$\begin{array}{l}\text { Talked with dr. on screenings } \\
\mathrm{X}_{(7)}^{2}=41.38(\mathrm{p}<.001) ; 73.7 \%\end{array}$}} \\
\hline & & & & \\
\hline & OR $[95 \% \mathrm{CI}]$ & $\mathrm{p}$-value & OR $[95 \% \mathrm{CI}]$ & $\mathrm{p}$-value \\
\hline Cancer Caregiver (Yes) & $1.90[.989,3.632]$ & 0.054 & $2.10[1.095,4.014]$ & $0.025^{*}$ \\
\hline Family History (Yes) & 1.05. $[.635,1.735]$ & 0.851 & $1.21[.706,2.063]$ & 0.493 \\
\hline Age $(>=40)$ & $2.75[1.530,4.942]$ & $<0.001 * *$ & $2.52[1.311,4.828]$ & $<0.006^{* *}$ \\
\hline Marital (married) & $2.57[1.303,5.077]$ & $0.006^{*}$ & $2.45[1.135,5.268]$ & $0.022 *$ \\
\hline Education $($ college + ) & $0.95[.464,1.953]$ & 0.894 & $1.06[.497,2.275]$ & 0.875 \\
\hline Gender (Male) & $1.47[1.469, .890]$ & 0.133 & $1.22[.717,2.074]$ & 0.464 \\
\hline Country (Asia) & $1.56[.937,2.612]$ & 0.087 & $1.40[.811,2.410]$ & 0.228 \\
\hline
\end{tabular}


communications specifically. Relevant results from previously published articles were integrated.

Current data show primary cancer caregivers were more likely to report doctor recommended screenings $(56.7 \%$ vs. $31.8 \%)$, communicated with doctor about screenings (48.3\% vs. $23.4 \%$ ), scored higher on cancer knowledge and warning signs, perceived higher cancer knowledge levels and screening norms (all $\mathrm{p}<.05$ ). Chinese adults with family cancer history were more likely to communicate with doctors about screenings $(32.2 \%$ vs. $21.5 \%$; $<.05)$ and perceived higher cancer risk (2.78 vs. 2.53; $\mathrm{p}=.003)$, lower health status (3.14 vs. 3.46; $\mathrm{p}=.001)$ and lower screening barriers (10.39 vs. 11.19; $\mathrm{p}=.011$ ). After controlling for demographics and country, regression analyses showed that caregiver experience was still a significant predictor on cancer communication with doctors $(\mathrm{OR}=2.1)$. Yet, the significant relationship of talk with doctors on screenings among those with family history disappeared.

Current study found that experience of being a primary cancer caregiver to a family member was significant to initiate communications with doctors about screening. This could be resulted from the increased awareness and knowledge towards cancer prevention, which were supported with current data and previous studies (Elangovan, et al, 2016; Polek et al., 2016). To shed some lights on the comparisons and findings, the previously published results found that Taiwan participants were more likely to have been a cancer caregiver (22.9\% vs. $10.4 \%$; $\mathrm{p}=0.001$ ) (Hou and Cao, 2019). In the specific analyses by country, Taiwan participants also scored higher on both objective cancer education knowledge scales: CSKT (mean of 6.13 vs. 5.52; $<<0.001$ ) and CWST (mean of 6.80 vs. 5.38; $\mathrm{p}<0.001)$. Taiwan participants also rated higher on perceived (subjective) cancer knowledge (3.09 vs. 2.59; $\mathrm{p}<0.001$ ), and endorsed higher on CSBS-Norms-3 (mean of 11.67 vs. 10.82; $<<0.001$ ) (Hou and Cao, 2019). The higher knowledge scores and more positive beliefs among Taiwan participants were consistent to the findings of the higher knowledge and beliefs found among primary caregivers in the current finding. The higher portion of caregiver from the Taiwan participants might partially contribute to these higher scores among the caregiver group. Nevertheless, the current regression analyses did included country as a co-variate, and still found caregiver was a significant predictor towards talking to doctors about cancer screenings.

However, attentions should be given to those with family cancer history. Current results showed there were no significant differences of family history on cancer communications with doctors, doctors recommending screenings, nor cancer screening knowledge or related beliefs. Potential factors that might contribute to the non-significant findings could be either doctors didn't ask or patients didn't mention about family history, or other communication related barriers. More studies should also examine reasons why Chinese with family cancer history perceived higher cancer risk and lower health status, yet still had the same low communication rate with doctors about screenings.

Doctor recommendation is one of the most consistent predictors of adherence to screening cancer screening (Jih et al., 2018). Consistent with the study hypotheses and recommended age-appropriate screening guidelines, current data showed that doctors were 2.45-2.52 times more likely to recommend screenings to those who were at older age ( $>=40$ years) or married. The previously published study which examined cancer knowledge by age provide some insights on potential underlying reasons (Hou and Liu, 2018b). While there were no significant differences between those who were older ( $>=40$ years) versus younger ( $<40$ years) on the objective cancer knowledge tests (CSKT and CWST), subjective knowledge was higher among older Chinese adults (younger.2.44 vs. older 3.05, p<.001) (Hou and Liu, 2018b). Older Chinese adults were also more likely to identify cancer warning signs correctly, while younger adults were more likely to identify false warning signs correctly. Studies have found that subjective knowledge to be a significant predictor on health behaviors (Hou and Liu, 2018b), thus the higher subjective knowledge among older Chinese participants might played role to the increased patient-doctor communication seen among older participants in the current findings.

The overall low doctor-patient cancer screening communication rate in the current finding is alarming. Existing studies show Chinese immigrants may face not only language barriers, but also cultural and other issues during the communication process (Jih et al., 2018; Kwok et al., 2011). Mixed methods research (MMR) which uses both quantitative and qualitative approaches to exam doctor-patient cancer communication might provide deepened and nuanced insights on some of the underlying reasons and barriers.

Study is limited to a convenience sample among faith-based Chinese adults whom self-selected to participated in the study. In addition, study sample consisted of middle-age Chinese adults mostly highly educated yet with English as their second language, thus results should be interpreted with such sample characteristics in mind, and not generalizing to other Chinese adult groups. The mixing of US churches with the Chinese church overseas participants, along with a deeper and specific examination of the current study focus provide a unique opportunity to further explore the complex relationships among doctor-patient communication and the role of cancer caregiver and family history.

Although sample size calculation showed sufficient power $(80 \%)$ to detect meaningful differences between groups, it should be noted that only a relative small proportion of the current sample $(17 \%)$ reported having been a primary cancer caregiver $(n=63)$. In addition, current study used one yes/no question to identify primary cancer caregiver experience. Additional dimensions related to the caregiver experience, such as lengths of time as a caregiver, might need to be considered in future research.

One strength of the current study was using items to capture the two-way doctor-patient communication dimensions, "Dr. recommended screenings" and "Talked to doctors about cancer screenings." The current study compared own doctor-patient cancer screening

Asian Pacific Journal of Cancer Prevention, Vol 22 
communication by the role of primary cancer caregiver and cancer family history. Future research could further exam the context or scenarios of a primary cancer caregiver or those with a family cancer history talking to doctor about a family cancer communication, instead of cancer communication about own case.

\section{Practical Implications}

The current study is among the first to note the significant relationship of primary cancer caregiver experience on increased cancer screening communications with doctors among Chinese church-going adults. Although data show caregivers were more likely to proactively communicate with doctors on screening, findings showed alarming finding that there was no significant difference on doctor's screening recommendations by family cancer history. This could possibly be the fact that not all individuals with family cancer history might need more frequent screenings. However, findings also point to the potential missed opportunities for needed screening referrals and the critical need of doctor training for culturally sensitive communication and screening this higher risk group (Lunsford et al., 2018; Rose, et al., 2001).

In conclusion, current study calls attentions to primary cancer caregiver experience, family history, age, and marital factors when designing tailored doctor-patient cancer communication programs among Chinese immigrants. Given the alarming overall low rate of doctor-patient cancer communication and high reported family cancer history (54\%) among the current the church-going highly educated Chinese study sample, study shed light on the potential to further exam how primary caregiver experience might serve as an active engagement factor to promote positive doctor-patient cancer screening communications.

Future studies using mixed-methods research (MMR) study designs are encouraged to provide nuanced insight on how the different dimensions of the caregiver experience, doctor-patient cancer screening communication, and how their experience might shape own cancer screening decisions. Similarly, MMR studies are recommended to exam concerns doctors might have when deciding whether or when to recommend screening for Chinese patients with family cancer history.

\section{Statement conflict of Interest}

The authors declare there is no conflict of interest.

\section{References}

Allen JD, Pérez JE, Tom L, et al (2014). A pilot test of a Churchbased intervention to promote multiple cancer-screening behaviors among latinas. J Cancer Educ, 29, 136-43.

American Cancer Society (2016). Report: wide variation in cancer rates in Asian American/Native Hawaiian/Pacific Islanders. Available from: http://pressroom.cancer.org/ AANHPICancerStats2016.

Anusha P, Nagarathnam M (2016). Assessment of knowledge on colorectal cancer among caregivers at tertiary care hospital, Andhra Pradesh. IOSR J Nurs Health Sci, 5, 52-61.

Centers for Disease Control and Prevention. (2019). Family Health History and Cancer. https://www.cdc.gov/cancer/ family-health-history/index.htm.

Chiang CJ, Lo WC, Yang YW, et al (2016). Incidence and survival of adult cancer patients in Taiwan, 2002-2012. J Formos Med Assoc, 115, 1076-88.

Claus E, Risch N, Thompson W (1991). Genetic analysis of breast cancer in the cancer and steroid hormone study. $\mathrm{Am}$ J Human Genett, 48, 232-42.

Colon-Otero G, Albertie M, Rodriguez J, et al (2014). A Churchbased, Spanish-language community education breast health program increases awareness and utilization of breast diagnostic services among hispanics. J High Educ Outreach Engagem, 18, 43-60,

Elangovan V, Rajaraman S, Basumalik B, Pandian D (2016). Awareness and perception about cancer among the public in Chennai, India. J Glob Oncol, 3, 469-79.

Emerson JS, Reece MC, Levine RS, Hull PC, Husaini BA (2009). Predictors of new screening for African American men participating in a prostate cancer educational program. J Cancer Educ, 24, 341-5.

Fletcher RH, Lobb R, Bauer MR, et al (2007). Screening patients with a family history of colorectal cancer. J Gen Intern Med, 22, 508-13.

Griffith KA, Passmore SR, Smith D, Wenzel J (2012). African Americans with a family history of colorectal cancer: Barriers and facilitators to screening. Oncol Nurs Forum, 39, 299-306.

Holt CL, Wynn TA, Litaker MS, et al (2009). A comparison of a spiritually based and non-spiritually based educational intervention for informed decision making for prostate cancer screening among church-attending African-American men. Urol Nurs, 29, 249.

Hou S (2007). Cancer Screening Belief Scale - Chinese version (CSBS-C): Instrument development and validation among a Chinese worksite population. Calif J Health Promot, 5, $79-88$.

Hou S (2010). Understand barriers and explore strategies to encourage preventive health service utilization among Chinese older adults. $J$ Ga Public Health Assoc, 5, 24-33.

Hou S (2016a). Cancer screening beliefs, source of health information, and recommended eHealth communication strategies among church-based Chinese adults. $J$ Cancer Educ, 31, 38-9.

Hou S (2016b). Demographics and cancer screening beliefs on regular annual health exam among church-based Chinese adults. $J$ Cancer Educ, 31, 39.

Hou S (2016c). Subjective and objective cancer screening knowledge among white- and blue-collar Chinese middleand older-age working adults. $J$ Cancer Educ, 33, 167-73.

Hou S, Cao X (2018a). A systematic review of promising strategies of faith-based cancer education and lifestyle interventions. J Cancer Educ, 33, 1161-75

Hou S, Cao X (2019). A cross-sectional study of cancer knowledge and beliefs among faith-based Chinese in the USA versus Taiwan. J Prev Med Public Health, 52, 60-5.

Hou S, Chen PH (2004). Home-administered fecal occult blood test for colorectal cancer screening among worksites in Taiwan. Prev Med, 38, 78-84.

Hou S, Fernandez M, Baumler E, Parcel GS, Chen P (2003). Correlates of cervical cancer screening among women in Taiwan. Health Care Women Int, 24, 384-98.

Hou S, Hou PH, Hou HS (2014). The impact of a colorectal cancers worksite screening intervention on knowledge, screening beliefs, and uptakes among middle- and older-age employees in Taiwan. J Cancer Educ, 29, 12-3.

Hou S, Liu L (2018b). Objective and subjective cancer knowledge among faith-based Chinese adults. Int Quarterly $J$ Community Health Educ, 38, 47-56. 
Jih J, Nguyen MP, Ly I, et al (2018). The role of physician recommendation in colorectal cancer screening receipt among immigrant Chinese Americans. J Immigr Minor Health, 20, 1483-9.

Kwok C, Koo FK, D'Abrew N, White K, Roydhouse JK (2011). East meets West: A brief report of a culturally sensitive breast health education program for Chinese-Australian women. $J$ Cancer Educ, 26, 540-6.

Lee C-Y, Lee Y, Wang L-J, et al (2017). Depression, anxiety, quality of life, and predictors of depressive disorders in caregivers of patients with head and neck cancer: A sixmonth follow-up study. J Psychol Res, 100, 29-34.

Lee S, Chen L, Jung MY, Baezconde-Garbanati L, Juon HS (2014). Acculturation and cancer screening among Asian Americans: role of health insurance and having a regular physician. J Community Health, 39, 201-12.

Lin JY, Diwanji TP, Snider JW, Knight N, RegineWF (2016). Cancer screening patterns and concerns in caregivers of patients undergoing radiation therapy. J Oncol Pract, 12, e405-12.

Longacre ML, Galloway TJ, Parvanta CF, Fang CY (2015). Medical communication-related informational need and resource preferences among family caregivers for head and neck cancer patients. J Cancer Educ, 30, 786-91.

Lunsford NB, Sapsis KF, Smither B, et al (2018). Young women's perceptions regarding communication with healthcare providers about breast cancer, risk, and prevention. $J$ Womens Health, 27, 162-70.

Nasiriani PK, Motevasselian MM, Farnia PF, Shiryazdi MS, Khodayarian PM (2017). The effect of telephone counseling and education on breast cancer screening in family caregivers of breast cancer patients. Intl J Community Based Nurs Midwifery, 5, 306-16.

Nipp R, El-Jawahri A, Fishbein J, et al (2016). Factors associated with depression and anxiety symptoms in family caregivers of patients with incurable cancer. Ann Oncol, 27, 1607-12.

Peterson EB, Ostroff JS, DuHamel KN, et al (2016). Impact of provider-patient communication on cancer screening adherence: A systematic review. Prev Med, 93, 96-105.

Polek C, Hardie T (2016). In Asian Americans, is having a family member diagnosed with cancer associated with fatalistic beliefs?. Asia Pac J Oncol Nurs, 3, 192-8.

Ramsey SD, Yoon P, Moonesinghe R, Khoury M (2006). Population-based study of the prevalence of family history of cancer: Implications for cancer screening and prevention. Genet Med, 8, 571-5.

Rose PW, Watson E, Yudkin P, et al (2001). Referral of patients with a family history of breast/ovarian cancer-GPs' knowledge and expectations. Fam Pract, 18, 487-90.

Salimzadeh H, Bishehsari F, Delavari A, et al (2016). Cancer risk awareness and screening uptake in individuals at higher risk for colon cancer: a cross-sectional study. BMJ Public Health, 6, e013833.

Schwingel A, Gálvez P (2016). Divine interventions: Faith-based approaches to health promotion programs for latinos. J Relig Health, 55, 1891-1906.

Sentell TL, Tsoh JY, Davis T, Davis J, Braun KL (2015). Low health litera $\neg \mathrm{cy}$ and cancer screening among Chinese Americans in Califor $\neg$ nia: a cross-sectional analysis. BMJ Open, 5, e006104.

Sklenarova H, Krümpelmann A, Haun MW, et al (2015). When do we need to care about the caregiver? Supportive care needs, anxiety, and depression among informal caregivers of patients with cancer and cancer survivors. Cancer, 121, 1513-19.

Warapornmongkholkul A, Howteerakul N, Suwannapong N, Soparattanapaisarn N (2018). Self-efficacy, social support, and quality of life among primary family-member caregivers of patients with cancer in Thailand. J Health Res, 32, 111-22.

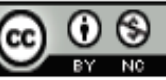

This work is licensed under a Creative Commons AttributionNon Commercial 4.0 International License. 